direction was not quantified.

Perhaps of greatest ecological significance is the demonstration that when the fir was in the shade, there was a considerable increase in the amount of carbon that it received from the birch. Most forest trees spend the early part of their lives as seedlings in the gloom of the forest floor. If, as indicated by this work, the direction of flux is determined by the carbon status of the recipient, the carbon economy of this shaded understorey will be subsidized by fully illuminated overstorey plants, through pathways provided by their fungal symbionts. The extent to which this subsidy might contribute to the ability of young trees to survive in shaded environments should clearly now be examined, because persistence under, and recruitment into, the canopy determines the equilibrium of the forest community. Moreover, certain plants that live on the forest floor notably in the family Monotropaceae totally lack chlorophyll, so they receive all of their carbon from photosynthesizing trees through mycorrhizal connections ${ }^{1}$. This illustrates the potential of these processes for sustaining receiver plants.

The observation that carbon is transferred between green plants will stimulate us to examine forest ecosystems from a fresh standpoint. It indicates that we should place less emphasis on competition between plants, and more on the distribution of resources within the community. If mycorrhizal colonization results in an equalization of resource availability, as suggested by this and a number of microcosm studies, it would be expected to reduce dominance of aggressive species, so promoting coexistence and greater biodiversity.

Since Clements ${ }^{8}$ introduced the concept of the community as a 'superorganism', ecologists have espoused the abstract idea that all components of stable ecosystems are interdependent. Simard et al. ${ }^{4}$ show the physical presence of interconnections between individuals in the forest ecosystem, and expose some of the likely consequences of their formation. The challenge now is to quantify further the contribution of these fungal linkages to the maintenance of biodiversity and stability in terrestrial ecosystems.

David Read is in the Department of Animal and Plant Sciences, University of Sheffield, Alfred Denny Building, Sheffield S10 2TN, UK.

1. Smith, S. E. \& Read, D. J. Mycorrhizal Symbiosis (Academic, London, 1996).

2. Remy, W., Taylor, T. N., Haas, H. \& Kerp, H. Proc. Natl Acad. Sci. USA 91, 11841-11843 (1994).

3. Simon, L., Bousquet, J., Levesque, R. C. \& Lalonde, M. Nature 363, 67-69 (1993).

4. Simard, S. W. et al. Nature 388, 579-582 (1997).

5. Molina, R., Massicotte, H. \& Trappe, J. in Mycorrhizal

Functioning (ed. Allen, M. F.) 357-423 (Chapman \& Hall, London, 1992).

6. Perry, D. A., Amaranthus, M. P., Borchers, J. G. \& Brainerd, R. C. BioScience 39, 230-237 (1989).

7. Newman, E. I. Adv. Ecol. Res. 18, 243-270 (1988).

8. Clements, F. Carnegie Inst. Wash. Publ. 242, 1-512 (1916)
Fluid dynamios

\section{A fractal world of cloistered waves}

Peter G. Baines

verybody is familiar with waves on the

- surface of the ocean. Waves longer - than about $1.7 \mathrm{~cm}$ are called gravity waves, because gravity (rather than surface tension) provides the principal restoring force that makes them possible. Less familiar are the similar waves that occur where there is an interface between two fluids of different densities, the lighter above the heavier. And if the density of an incompressible fluid decreases continuously upwards, waves may occur throughout the body of the fluid. Such waves are termed internal gravity waves. They permeate both the atmosphere and the ocean, and are very important for their internal dynamics on small to medium scales, where they transport momentum and cause local mixing. They may also exist in closed containers, where they converge on

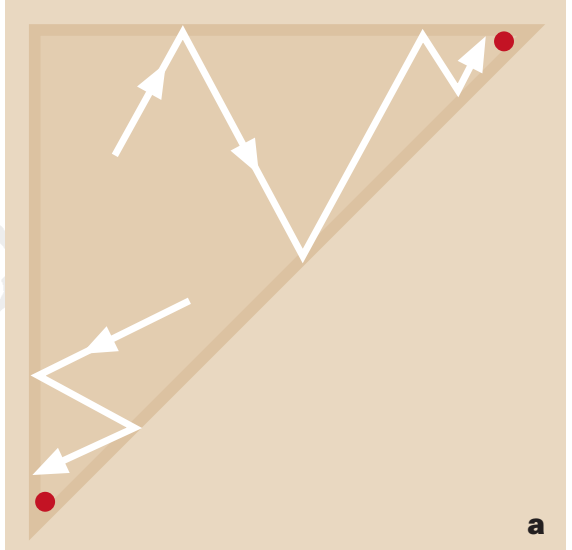

'attractors' - closed curves determined by the geometry of the container - and then propagate energy along them (Fig. 1). These bizarre properties have been verified by experiments by Maas et al. ${ }^{1}$, described on page 557 of this issue.

The propagation properties of internal gravity waves are much stranger than those of sound or electromagnetic waves. In particular, there is a maximum frequency that the waves may have (the buoyancy frequency); the direction in which they transmit energy (the group velocity) is perpendicular to the direction in which they propagate (the phase velocity); and in a uniformly stratified fluid, all waves of any given frequency propagate at the same angle to the horizontal, regardless of their length or structure. This last property means that if a density-strati-

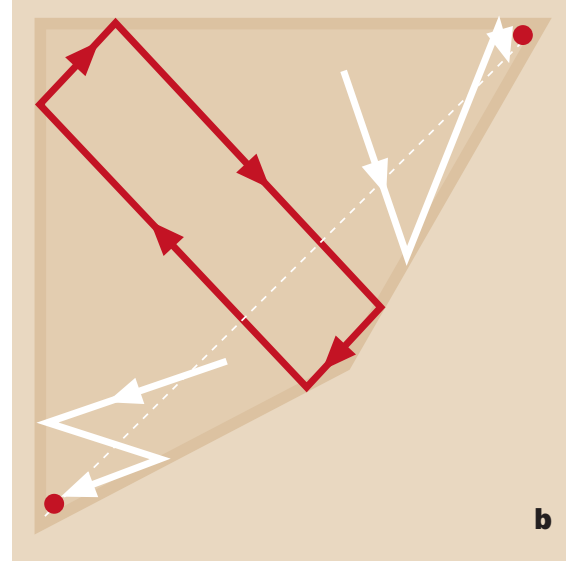

Figure 1 Models of attractor. Internal gravity waves in a fluid carry energy at an angle to the vertical that depends on their frequency. For low frequencies the rays are nearly horizontal; near the buoyancy frequency (the upper limit) the rays approach the vertical. a, In a triangular container, rays steeper than the diagonal wall go towards the right-hand vertex, and those at a shallower angle go to the bottom vertex - these two points are attractors for all internal waves. b, In a quadrilateral, rays at angles in between those of the two sloping sides converge on interior attractors. A simple example, for a particular frequency, is shown. For most of these intermediate frequencies, the attractors are much more complicated, including many circuits and having a fractal dependence on frequency.

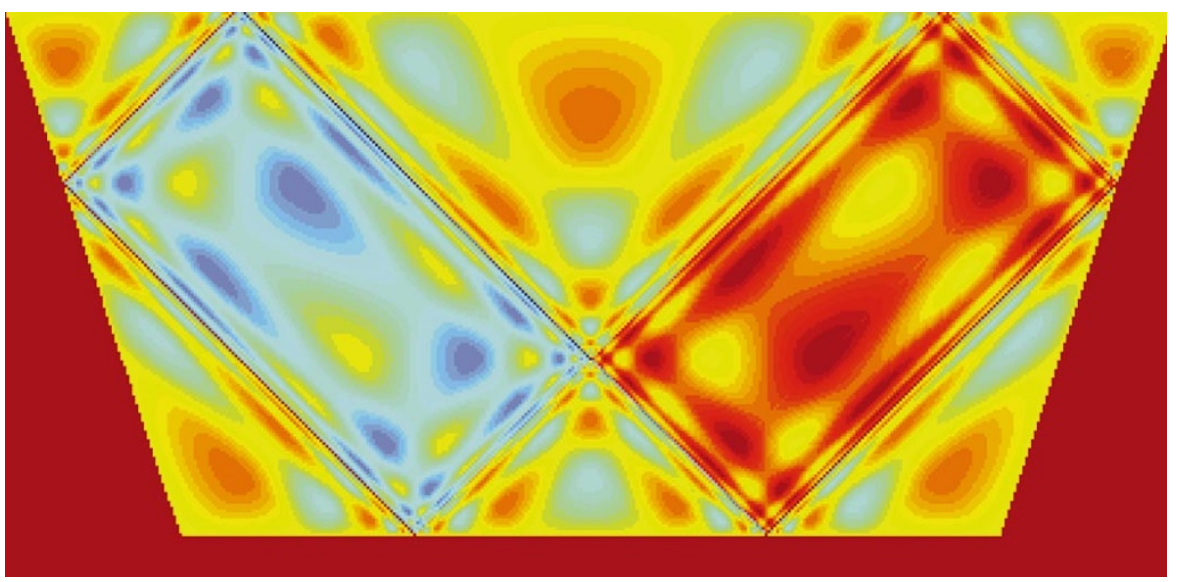

Figure $2 \mathrm{~A}$ wave in a bucket. For a range of wave frequencies, the fluid flow field marks out an attractor that consists of two rectangular boxes. 
fied fluid is forced at a frequency less than the buoyancy frequency, all the wave energy must propagate at these fixed directions. This must also apply to the waves after they reflect from solid boundaries, regardless of the orientation of the reflecting boundary. If the waves happen to be generated inside a closed container that has an irregular shape, a complex and apparently messy field of motion can result. Further, the mathematical problem of determining these properties is ill posed, and is equivalent to solving a hyperbolic partial differential equation with elliptic boundary conditions. The 'solution' is very sensitive to the geometry of the wave rays (or characteristics) inside the container. This system has an important analogue: inertial waves (due to the Coriolis force) in rotating bodies of fluid, which have similar properties.

In geophysical fluids, the principal manifestations of the unidirectional property of internal wave propagation at a particular frequency are the internal tides in the ocean. These are internal gravity waves of tidal period, generated by the oscillatory advection of the stratified ocean over topographical features on the floor of the ocean by the surface tide forced by the Sun and the Moon. Here the internal tide propagates away from the source regions into the vastness of the ocean on rays at the required vertical angle. This mathematical problem is (mostly) well posed $^{2}$, and these properties have been experimentally verified ${ }^{3}$ and well known for at least three decades. Field observations in the Bay of Biscay ${ }^{4}$ have confirmed the essential features of the wave propagation theory.

For some time, then, the basic physics of these processes in open geometries has been regarded as reasonably well known, but, in contrast, the problem of waves in irregularly shaped closed containers was thought to be too complicated and, partly in consequence, not very important or interesting. However, the growth of the theory of chaos and fractals in recent years has reawakened interest in the problem from a mathematical viewpoint. In particular, a few years ago Maas and $\mathrm{Lam}^{5}$ showed theoretically that, in simple containers of trapezium shape, wave energy inside the container at a single frequency may converge to an 'attractor', which consists of a closed line figure that may be as simple as a single parallelogram. The attractor is purely determined by the geometry of the container, and the wave energy converges on it and then propagates around it, as can clearly be seen in the new results ${ }^{1}$. The shape of this attractor changes continuously with the frequency of the waves over some ranges of values, and varies fractally over others (this has similarities to the scarred wavefunctions of quantum $\operatorname{chaos}^{6}$ ).

Where such attractors occur, the motion cannot be represented as a sum (or Fourier series) of wave modes to simplify the description of the motion, as such modes do not exist. This comes as quite a shock to most physicists, who are accustomed to representing wave motions in closed containers of all types in terms of a sum of stationary-wave modes. It is not safe to assume, for example, that internal wave motion in a given densitystratified lake can be represented in terms of a sum of modes.

The experimental confirmation now presented by Maas et al. shows that the simplifying concept of the attractor is relevant and useful, and it should provide a stimulus for further theoretical developments and a search for more practical applications. Possibilities for the latter include transverse wave motions in fjords, and internal tides in topographically complex regions of the ocean. There has been a resurgence of interest in internal tides, as they have recently become detectable by satellite altimetry that measures changes in height of the sea surface, and are more prominent and ubiquitous than thought previously. But the most significant applications may lie in the rotating analogue that I mentioned earlier; here there are many possibilities, perhaps the most intriguing being the likely presence of these effects in the liquid outer core of the slightly nonspherical Earth.

Peter G. Baines is at the CSIRO Division of

Atmospheric Research, Aspendale 3195, Australia.

1. Maas, L. R. M., Benielli, D., Sommeria, J. \& Lam, F.-P. A. Nature 388, 557-561 (1997).

2. Baines, P. G. Deep-Sea Res. 29, 307-338 (1982)

3. Mowbray, D. E. \& Rarity, B. S. H. J. Fluid Mech. 28, 1-16 (1967).

4. Pingree, R. D. \& New, A. L. Deep-Sea Res. 36, 735-758 (1989); J. Phys. Oceanogr. 21, 28-39 (1991).

5. Maas, L. R. M. \& Lam, F.-P. J. Fluid Mech. 300, 1-41 (1995).

6. Heller, E. J. Nature 380, 583-584 (1996).

\section{IKB kinase all zipped up}

\section{Alain Israël}

N $\mathrm{F}-\kappa \mathrm{B}$ is a transcription factor that plays a central role in the immune and inflammatory response, and is also involved in controlling the expression of human immunodeficiency virus. A few years after this factor was identified ${ }^{1}$, it became clear that its activity is controlled by a previously unknown mechanism. In most cells, NF-кB is retained in the cytoplasm in an inactive form by the inhibitory protein IкB. Following certain extracellular stimuli (the most frequently studied being tumour-necrosis factor (TNF), interleukin-1 (IL-1), phorbol myristate acetate (PMA) or lipopolysaccharide), NF- $\kappa \mathrm{B}$ dissociates from I $\mathrm{B}$, translocates to the nucleus and activates its target genes (see Fig. 1, and ref. 2 for review).

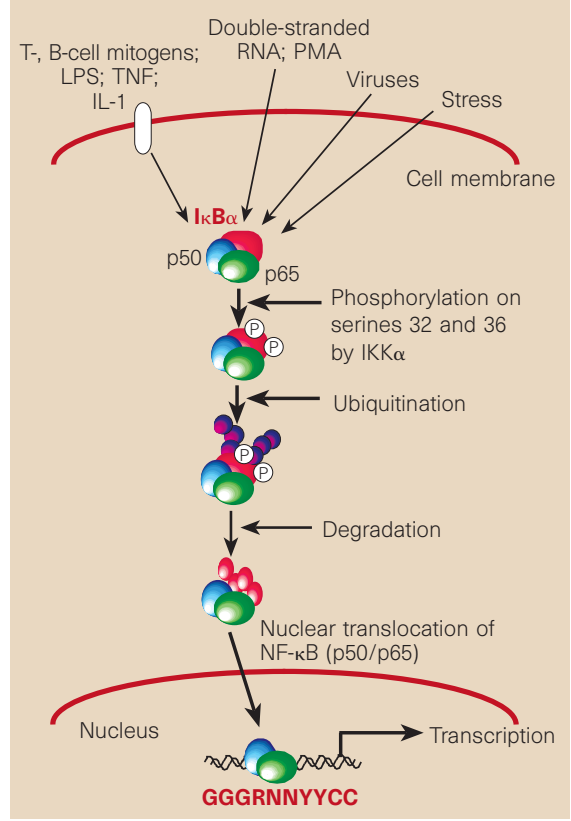

Even before the first member of the NF$\kappa \mathrm{B}$ family was cloned, it was recognized that the association of I $\kappa \mathrm{B}$ with NF- $\kappa \mathrm{B}$ is controlled by phosphorylation, and thus the search for the protein kinase responsible began. On page 548 of this issue ${ }^{3}$, DiDonato et al. report the purification and cloning of a cytokine-responsive IкB kinase which might put an end to this long and frustrating search.

In 1990, Ghosh and Baltimore ${ }^{4}$ demonstrated that treatment of a partially purified $\mathrm{NF}-\kappa \mathrm{B} / \mathrm{I} \kappa \mathrm{B}$ complex by certain kinases could induce dissociation of the complex and activation of NF- $\kappa \mathrm{B}$ DNA-binding activity. Cloning ${ }^{5}$ of the complementary DNA encoding I $\mathrm{I} B \alpha$, one of the three forms

Figure 1 The NF- $\kappa$ B signalling pathway (after a graphic by J. C. Epinat). The pathway is activated by various stimuli, some of which may also use alternative pathways. The stimuli include $\mathrm{T}$ - and B-cell mitogens, lipopolysaccharide (LPS), tumour-necrosis factor (TNF) and interleukin-1 (IL-1), double-stranded RNA or phorbol myristate acetate (PMA), infection by certain viruses, or stress (such as irradiation with ultraviolet light). The ensuing signalling cascade results in phosphorylation of the inhibitory protein, I $\kappa$ B $\alpha$, by IKK $\alpha$, the protein kinase which DiDonato et al. ${ }^{3}$ have now isolated. I $\mathrm{B} \alpha$ is then ubiquitinated and degraded by proteasomes, and NF- $\kappa$ B translocates into the nucleus to modulate gene transcription. The consensus binding site for NF- $\kappa B$ is shown ( $R$, purines; $Y$, pyrimidines; $\mathrm{N}$, any nucleotide). The five members of the NF- $\kappa$ B family can form homo- or heterodimers (p50 and p65 are the constituents of NF- $\kappa B$ activity). IKK $\alpha$ activation by stimuli other than TNF, IL-1 and PMA has not yet been investigated. 